\title{
On the Bombieri-Korobov estimate for Weyl sums
}

by

\author{
Scott T. PARSELl (Indianapolis, IN)
}

1. Introduction. In studying Waring's problem and other additive questions involving $k$ th powers, a fundamental role is played by estimates for the exponential sum

$$
f(\boldsymbol{\alpha})=f_{k}(\boldsymbol{\alpha} ; P)=\sum_{1 \leq x \leq P} e\left(\alpha_{k} x^{k}+\cdots+\alpha_{1} x\right),
$$

where we have written $e(y)=e^{2 \pi i y}$. When $\alpha_{k}$ lies in a suitable set of minor arcs, methods based on Weyl differencing and Vinogradov's mean value theorem yield non-trivial upper bounds for $|f(\boldsymbol{\alpha})|$. In Vinogradov's method, which is more effective for larger $k$, one obtains minor-arc estimates for $|f(\boldsymbol{\alpha})|$ from bounds for the mean values

$$
J_{s, k}(P)=\int_{[0,1]^{k}}|f(\boldsymbol{\alpha})|^{2 s} d \boldsymbol{\alpha} .
$$

When $s$ is an integer, one sees by orthogonality that $J_{s, k}(P)$ is the number of solutions of the system of equations

$$
x_{1}^{i}+\cdots+x_{s}^{i}=y_{1}^{i}+\cdots+y_{s}^{i} \quad(1 \leq i \leq k)
$$

with $x_{j}, y_{j} \in[1, P] \cap \mathbb{Z}$. By using a $p$-adic iteration method, one may obtain estimates of the shape

$$
J_{s, k}(P) \ll P^{2 s-k(k+1) / 2+\eta(s, k)+\varepsilon},
$$

where the sharpest values for $\eta(s, k)$ are due to the repeated efficient differencing technology of Wooley [9]. We observe that the mean value $J_{s, k}(P)$ is well-defined for non-integral $s$ as well. Although it then lacks an interpretation as the number of solutions of (1.1), estimates of the shape (1.2) follow by interpolation via Hölder's inequality.

2000 Mathematics Subject Classification: 11L07, 11L15, 11P05, 11P55.

Key words and phrases: Weyl sums, estimates on exponential sums, Waring's problem, applications of the Hardy-Littlewood method. 
When $\alpha_{k}$ has a rational approximation with relatively large denominator, arguments of Bombieri [2] and Korobov [5] produce estimates of the shape $|f(\boldsymbol{\alpha})| \ll P^{1-\sigma(k)+\varepsilon}$, where $\sigma(k)$ is determined by the available mean value estimates. This technique, when combined with the results of Wooley [11] on smaller moduli, yields the sharpest currently available Weyl exponents for $k \geq 14$. The main purpose of this note is to provide an alternative method for obtaining the results for larger moduli. In contrast to the arguments of Bombieri and Korobov, our derivation makes use of a standard Weyl shift and the large sieve in a manner familiar to additive number theorists. In the process, we make an additional observation that yields modest improvements in the existing Weyl exponents. In the standard arguments (see for example $[1$, Chapter 4$]$ and $[7$, Chapter 5]) the Weyl shift initially makes use of a rather arbitrary set of integers within the set $[1, P]$, which is later specialized in order to achieve the spacing condition required for an application of the large sieve. In our argument, we retain the arbitrary nature of the set and simply split the relevant sum into a bounded number of sub-sums, each having the desired property. Moreover, because of an interchange in the order of summation, we obtain an effect similar to that of Wooley [11, Lemma 4], in that the main mean values relevant to our argument involve variables restricted to this set. Thus we can potentially gain an advantage by using a convenient set of integers. The same principle may be applied to the arguments of [1] and [7] by interchanging the roles of the variables. We first state a result essentially equivalent to Theorem 8 of Bombieri [2].

Theorem 1.1. Let $\mathcal{A} \subseteq[1, P] \cap \mathbb{Z}$ with $|\mathcal{A}|=Q$, and write $J_{s, k}(\mathcal{A})$ for the number of solutions of (1.1) with $x_{j}, y_{j} \in \mathcal{A}$. If $\left|q \alpha_{k}-a\right| \leq q^{-1}$ with $(q, a)=1$ and $r, s$, and $t$ are positive integers with $t \leq k-1$, then one has

$$
|f(\boldsymbol{\alpha})|^{2 r s} \ll Q^{-2 s}(\log 2 P)^{2 r s} H(q, P) P^{2 r s-r+k(k-1) / 2} J_{r / 2, t}(2 P) J_{s, k-1}(\mathcal{A}),
$$

where

$$
H(q, P)=\prod_{j=k-t}^{k-1}\left(1+q P^{-j}\right)\left(1+P^{k-j} q^{-1}\right) .
$$

We now describe an interesting special case of the theorem. Let

$$
\mathcal{A}(P, R)=\{n \in[1, P] \cap \mathbb{Z}: p \mid n, p \text { prime } \Rightarrow p \leq R\}
$$

denote the set of $R$-smooth numbers up to $P$, and let $U_{s, k}(P, R)$ denote the number of solutions of the system (1.1) with $x_{j}, y_{j} \in \mathcal{A}(P, R)$. When $R$ is a sufficiently small power of $P$, the technology of Wooley [13] yields estimates of the shape

$$
U_{s, k}(P, R) \ll P^{2 s-k(k+1) / 2+\Delta(s, k)+\varepsilon},
$$

where $\Delta(s, k)$ behaves essentially like $\eta(s, k)$ for large $k$ but may in fact be somewhat smaller for specific moderately-sized values of $k$. We sometimes 
refer to exponents $\eta(s, k)$ and $\Delta(s, k)$ satisfying (1.2) and (1.3) as admissible. The advantages of using smooth numbers would be much more significant if we were dealing with an incomplete system, but we can nevertheless expect some modest improvements.

COROLlary 1.2. Suppose that $\left|q \alpha_{k}-a\right| \leq q^{-1}$, where $P^{r} \leq q \leq P^{k-r}$ and $(q, a)=1$. Whenever $1 \leq r \leq k / 2$ and $\Delta(s, k-1)$ is an admissible exponent for (1.3), one has $|f(\boldsymbol{\alpha})| \ll P^{1-\mu(k)+\varepsilon}$, where

$$
\mu(k)=\frac{r-\Delta(s, k-1)}{2 r s} .
$$

One may now optimize over $r$ in conjunction with Lemma 4 of Wooley [11] to obtain new Weyl exponents. We give a description of these computations in $\S 4$.

2. Preliminaries. We begin by recalling a standard Weyl shift (see equation (5.23) of Vaughan [7]).

Lemma 2.1. Let $\mathcal{A} \subseteq[1, P] \cap \mathbb{Z}$ with $|\mathcal{A}|=Q$. Then

$$
|f(\boldsymbol{\alpha})| \ll Q^{-1} \log 2 P \sup _{\beta \in[0,1]} \sum_{y \in \mathcal{A}}|g(\boldsymbol{\alpha} ; \beta, y)|
$$

where

$$
g(\boldsymbol{\alpha} ; \beta, y)=\sum_{x=1}^{2 P} e\left(\sum_{i=1}^{k} \alpha_{i}(x-y)^{i}+\beta x\right) .
$$

We also need the following multi-dimensional version of the large sieve (see Vaughan [7, Lemma 5.3]).

Lemma 2.2. Suppose that $\Gamma \subseteq \mathbb{R}^{l}$ and that the sets

$$
R(\boldsymbol{\gamma})=\left\{\boldsymbol{\beta}:\left\|\beta_{j}-\gamma_{j}\right\|<\delta_{j}: 0 \leq \beta_{j}<1\right\}
$$

with $\gamma \in \Gamma$ are pairwise disjoint. Let $\mathcal{M}$ denote the set of integer l-tuples $\mathbf{m}$ with $1 \leq m_{j} \leq M_{j}$, and write

$$
S(\boldsymbol{\beta})=\sum_{\mathbf{m} \in \mathcal{M}} a(\mathbf{m}) e(\boldsymbol{\beta} \cdot \mathbf{m}) .
$$

Then

$$
\sum_{\boldsymbol{\gamma} \in \Gamma}|S(\gamma)|^{2} \ll \sum_{\mathbf{m} \in \mathcal{M}}|a(\mathbf{m})|^{2} \prod_{j=1}^{l}\left(M_{j}+\delta_{j}^{-1}\right) .
$$

Finally, we recall a result on the number of solutions of a diophantine inequality (see Bombieri [2, Lemma 3]).

LEMMA 2.3. If $\alpha$ is a real number with $|q \alpha-a| \leq q^{-1}$ for some integers $a$ and $q$ with $(q, a)=1$ and $m$ is a positive integer, then the number of 
solutions of the inequality

$$
\|m \alpha x+\beta\| \leq 1 / Y
$$

with $|x| \leq X$ and $x \in \mathbb{Z}$ does not exceed $(1+4 q / Y)(1+4 m X / q)$.

3. The alternative proof. In this section we obtain the Weyl-type estimates advertised in Theorem 1.1. Suppose that $\left|q \alpha_{k}-a\right| \leq q^{-1}$, where $(q, a)=1$, let $\mathcal{A}$ be a set of integers in $[1, P]$ with $|\mathcal{A}|=Q$, and let $r, s$, and $t$ be positive integers with $t \leq k-1$. Then by Lemma 2.1 and Hölder's inequality we have

$$
|f(\boldsymbol{\alpha})|^{r} \ll Q^{-1} L^{r} \sum_{y \in \mathcal{A}}\left|\sum_{x=1}^{2 P} e\left(\sum_{i=1}^{k} \alpha_{i}(x-y)^{i}+\beta x\right)\right|^{r}
$$

for some $\beta \in[0,1]$, where we have written $L=\log 2 P$. Hence there exist complex numbers $\zeta_{y}$ with $\left|\zeta_{y}\right|=1$ such that

$$
|f(\boldsymbol{\alpha})|^{r} \ll Q^{-1} L^{r} \sum_{y \in \mathcal{A}} \zeta_{y} \sum_{\mathbf{x} \in[1,2 P]^{r}} e\left(\sum_{i=1}^{k} \alpha_{i} S_{i}(\mathbf{x}, y)+\beta\left(x_{1}+\cdots+x_{r}\right)\right),
$$

where we have written

$$
S_{i}(\mathbf{x}, y)=\left(x_{1}-y\right)^{i}+\cdots+\left(x_{r}-y\right)^{i} .
$$

Now by interchanging the order of summation and using Hölder's inequality again we obtain

$$
|f(\boldsymbol{\alpha})|^{2 r s} \ll Q^{-2 s} L^{2 r s} P^{r(2 s-1)} \sum_{\mathbf{x} \in[1,2 P]^{r}}\left|\sum_{y \in \mathcal{A}} \zeta_{y} e\left(\sum_{i=1}^{k} \alpha_{i} S_{i}(\mathbf{x}, y)\right)\right|^{2 s} .
$$

We next observe that

$$
\sum_{i=1}^{k} \alpha_{i}(x-y)^{i}=(-1)^{k} \alpha_{k} y^{k}+\sum_{j=1}^{k-1} A_{j}(x) y^{j}+\sum_{i=1}^{k} \alpha_{i} x^{i},
$$

where

$$
A_{j}(x)=(-1)^{j} \sum_{i=j}^{k}\left(\begin{array}{l}
i \\
j
\end{array}\right) \alpha_{i} x^{i-j} .
$$

We therefore deduce that

$$
|f(\boldsymbol{\alpha})|^{2 r s} \ll \Upsilon \sum_{\mathbf{x} \in[1,2 P]^{r}}\left|\sum_{y \in \mathcal{A}} \zeta_{y} e\left((-1)^{k} r \alpha_{k} y^{k}+\sum_{j=1}^{k-1} B_{j}(\mathbf{x}) y^{j}\right)\right|^{2 s},
$$


where $\Upsilon=Q^{-2 s} L^{2 r s} P^{r(2 s-1)}$ and

$$
B_{j}(\mathbf{x})=(-1)^{j} \sum_{i=j}^{k}\left(\begin{array}{l}
i \\
j
\end{array}\right) \alpha_{i}\left(x_{1}^{i-j}+\cdots+x_{r}^{i-j}\right) .
$$

Let $b(\mathbf{n})$ denote the number of solutions of the system

$$
x_{1}^{l}+\cdots+x_{r}^{l}=n_{l} \quad(1 \leq l \leq t)
$$

with $\mathbf{x} \in[1,2 P]^{r}$, and write

$$
\gamma_{j}(\mathbf{n})=(-1)^{j} \sum_{i=j}^{k}\left(\begin{array}{l}
i \\
j
\end{array}\right) \alpha_{i} n_{i-j}
$$

Further, let $c(\mathbf{m})$ denote the number of solutions of the system

$$
y_{1}^{j}+\cdots+y_{s}^{j}=m_{j} \quad(1 \leq j \leq k-1)
$$

with $\mathbf{y} \in \mathcal{A}^{s}$, and let $\tilde{c}(\mathbf{m})$ denote the corresponding weighted count, where each solution is counted with weight

$$
w_{\mathbf{y}}=\zeta_{y_{1}} \cdots \zeta_{y_{s}} e\left((-1)^{k} r \alpha_{k}\left(y_{1}^{k}+\cdots+y_{s}^{k}\right)\right) \text {. }
$$

We then have

$$
|f(\boldsymbol{\alpha})|^{2 r s} \ll \Upsilon \sum_{\mathbf{n}, \mathbf{x}}\left|\sum_{\mathbf{m}, \mathbf{y}} w_{\mathbf{y}} e\left(\sum_{j=k-t}^{k-1} \gamma_{j}(\mathbf{n}) m_{j}+\sum_{j=1}^{k-t-1} B_{j}(\mathbf{x}) m_{j}\right)\right|^{2},
$$

where the summations are over $\mathbf{x}$ counted by $b(\mathbf{n})$ and $\mathbf{y}$ counted by $c(\mathbf{m})$. Write

$$
M_{1}=\sum_{j=1}^{k-t-1} j=\left(\begin{array}{c}
k-t \\
2
\end{array}\right) \quad \text { and } \quad M_{2}=\sum_{j=k-t}^{k-1} j=\left(\begin{array}{l}
k \\
2
\end{array}\right)-\left(\begin{array}{c}
k-t \\
2
\end{array}\right),
$$

and view $\mathbf{m}=\left(\mathbf{m}_{1}, \mathbf{m}_{2}\right)$ as an element of $\mathbb{Z}^{k-t-1} \times \mathbb{Z}^{t}$. Using Cauchy's inequality to isolate the contribution from the indices $j$ with $k-t \leq j \leq k-1$, we obtain

$$
|f(\boldsymbol{\alpha})|^{2 r s} \ll \Upsilon \sum_{\mathbf{n}} b(\mathbf{n}) P^{M_{1}} \sum_{\mathbf{m}_{1}}\left|\sum_{\mathbf{m}_{2}} \tilde{c}(\mathbf{m}) e\left(\sum_{j=k-t}^{k-1} \gamma_{j}(\mathbf{n}) m_{j}\right)\right|^{2} .
$$

Now for each $j$ with $k-t \leq j \leq k-1$ we have $\gamma_{j}(\mathbf{n})=d_{j} \alpha_{k} n_{k-j}+\varpi_{j}(\mathbf{n})$ for some integer $d_{j}$, where $\varpi_{j}(\mathbf{n})$ depends only on $n_{k-j-1}, \ldots, n_{1}$ and $\boldsymbol{\alpha}$. Hence we may successively apply Lemma 2.3 , starting with $j=k-1$, to deduce that the system of diophantine inequalities

$$
\left\|\gamma_{j}(\mathbf{n})-\gamma_{j}\left(\mathbf{n}^{\prime}\right)\right\|<P^{-j} \quad(k-t \leq j \leq k-1)
$$


has at most

$$
H=H(q, P) \ll \prod_{j=k-t}^{k-1}\left(1+q P^{-j}\right)\left(1+P^{k-j} q^{-1}\right)
$$

solutions $\mathbf{n}$ for every fixed $\mathbf{n}^{\prime}$. We may therefore split the sum over $\mathbf{n}$ into $H$ sub-sums over sets $\mathcal{N}_{1}, \ldots, \mathcal{N}_{H}$ with the property that for each $i$ the sets

$$
\left\{\boldsymbol{\beta} \in[0,1)^{t}:\left\|\gamma_{j}(\mathbf{n})-\beta_{j}\right\|<\frac{1}{2} P^{-j}: 0 \leq \beta_{j}<1\right\}
$$

corresponding to distinct $\mathbf{n} \in \mathcal{N}_{i}$ are pairwise disjoint. It therefore follows from Lemma 2.2 that for each $\mathbf{m}_{1}$ and each $i$ one has

$$
\sum_{\mathbf{n} \in \mathcal{N}_{i}}\left|\sum_{\mathbf{m}_{2}} \tilde{c}(\mathbf{m}) e\left(\sum_{j=k-t}^{k-1} \gamma_{j}(\mathbf{n}) m_{j}\right)\right|^{2} \ll \sum_{\mathbf{m}_{2}}|\tilde{c}(\mathbf{m})|^{2} \prod_{j=k-t}^{k-1} P^{j} .
$$

Notice that

$$
b(\mathbf{n})=\int_{[0,1]^{t}} g(\boldsymbol{\alpha})^{r} e(-\boldsymbol{\alpha} \cdot \mathbf{n}) d \boldsymbol{\alpha} \leq \int_{[0,1]^{t}}|g(\boldsymbol{\alpha})|^{r} d \boldsymbol{\alpha}=J_{r / 2, t}(2 P)
$$

for all $\mathbf{n}$, where $g(\boldsymbol{\alpha})=f_{t}(\boldsymbol{\alpha} ; 2 P)$. On inserting (3.2) into (3.1), we therefore obtain

$$
|f(\boldsymbol{\alpha})|^{2 r s} \ll Q^{-2 s} L^{2 r s} H(q, P) P^{r(2 s-1)+M_{1}+M_{2}} J_{r / 2, t}(2 P) \sum_{\mathbf{m}}|\tilde{c}(\mathbf{m})|^{2} .
$$

Moreover, one has $|\tilde{c}(\mathbf{m})| \leq c(\mathbf{m})$ and

$$
\sum_{\mathbf{m}} c(\mathbf{m})^{2}=J_{s, k-1}(\mathcal{A})
$$

so on noting that $M_{1}+M_{2}=\frac{1}{2} k(k-1)$ we find that

$$
|f(\boldsymbol{\alpha})|^{2 r s} \ll Q^{-2 s} L^{2 r s} H(q, P) P^{2 s r-r+k(k-1) / 2} J_{r / 2, t}(2 P) J_{s, k-1}(\mathcal{A}),
$$

and this completes the proof of Theorem 1.1.

In order to deduce Corollary 1.2, we take $t=r$ and $\mathcal{A}=\mathcal{A}(P, R)$ with $R$ a small power of $P$, so that $Q \asymp P$. By applying the argument of the proof of Vaughan [7, Lemma 5.1], one sees that $J_{r / 2, r}(2 P) \leq r$ !. Moreover, the hypothesis that $P^{r} \leq q \leq P^{k-r}$ with $r \leq k / 2$ shows that $H(q, P) \ll 1$. The corollary now follows immediately on substituting the estimate

$$
J_{s, k-1}(\mathcal{A})=U_{s, k-1}(P, R) \ll P^{2 s-k(k-1) / 2+\Delta(s, k-1)+\varepsilon} .
$$

One could also reverse the roles of the variables in the Weyl shift (see Wooley [11, Lemma 2]) and then attempt to take $r>t$ and inject smooth number technology into the analogue of $b(\mathbf{n})$, but this seems to produce inferior results. 
4. An application. As mentioned in the introduction, the flexibility arising from the set $\mathcal{A}$ in Theorem 1.1 permits modest improvements on the Weyl exponents calculated by Ford [3]. Our aim in this section is to briefly describe the ingredients involved in using Corollary 1.2 to carry out such computations. As a starting point for our mean value estimates, we recall that $J_{s, k}(P)$ and $U_{s, k}(P, R)$ exhibit diagonal behavior for $s \leq k+1$ (see Hua $\left[4\right.$, Lemma 5.4]). Hence the exponents $\eta(s, k)=\Delta(s, k)=\frac{1}{2} k(k+1)-s$ are admissible when $s \leq k+1$. We obtain further admissible exponents for (1.3) using the iterative method of Wooley [13].

LEMma 4.1. Suppose that $\Delta_{s}=\Delta(s, k)$ is an admissible exponent for (1.3). Given $j$ with $1 \leq j \leq k$, define $\phi_{j}=1 / k$ and for $J=j, \ldots, 2$, set

$$
\phi_{J-1}^{*}=\frac{1}{2 k}+\left(\frac{1}{2}+\frac{J(J-1)-2 \Delta_{s}}{4 k(k-J+1)}\right) \phi_{J}
$$

and $\phi_{J-1}=\min \left\{1 / k, \phi_{J-1}^{*}\right\}$. Then the exponent

$$
\Delta_{s+k}=\Delta_{s}\left(1-\phi_{1}\right)+k\left(k \phi_{1}-1\right)
$$

is also admissible.

Proof. This is a special case of [13, Lemma 6.1]. Here one has

$$
t=k, \quad k_{i}=k-i+1, \quad \tilde{r}_{J}=k-J+1, \quad \text { and } \quad \Omega_{J}=\frac{1}{2} J(J+1)
$$

in the notation of that lemma.

Note that the parameter $\phi_{1}$ depends on the number of differences $j$ (in addition to $s$ ), so for each $s$ we minimize over $1 \leq j \leq k$ to obtain the optimal value. As mentioned in [10], the methods of [13] also permit one to establish quasi-diagonal behavior for the system (1.1) restricted to smooth numbers. The fundamental relationship is embodied in the following lemma.

LEMMA 4.2. Let $l=\lfloor k / 2\rfloor$, and suppose that $r$ and $t$ are natural numbers satisfying $3 \leq r \leq k, 1 \leq t<2 l$, and $r+t \geq k$. If $v=s(1-t / 2 l)^{-1}$ and $0<\theta \leq 1 / r$ then one has

$$
U_{s+t, k}(P, R) \ll P^{(2 s+\omega(r, t, k)) \theta}\left(P^{t} U_{s, k}(Q, R)+P^{(t / 2)(2-r \theta)}\left(U_{v, k}(Q, R)\right)^{s / v}\right),
$$

where $Q=P^{1-\theta}$ and

$$
\omega(r, t, k)=\frac{1}{2}(r+t-k-1)(r+t-k) .
$$

Proof. This follows by applying the arguments of [13, Lemmas 4.1 and 4.2], within the argument of [10, Lemma 4.2], after adjusting the application of Hölder's inequality in the latter proof to accommodate a fractional moment.

If one has estimates of the shape $U_{s, k}(Q, R) \ll Q^{\lambda_{s}+\varepsilon}$, then the two terms in Lemma 4.2 are equal when 


$$
\theta=\frac{2\left(s \lambda_{v}-v \lambda_{s}\right)}{2 s \lambda_{v}+r t v-2 v \lambda_{s}}
$$

We take this value for $\theta$ whenever it does not exceed $1 / r$ and take $\theta=1 / r$ otherwise. As in the computations of Ford [3], we apply Lemmas 4.1 and 4.2 iteratively to obtain the best results. We may then apply the argument of Wooley [11, Theorem 2], using Corollary 1.2 in place of [11, Lemma 3], and optimize over $1 \leq r \leq k / 2$ to obtain new Weyl exponents. For larger $k$, our improvements are not significant, as the advantage of restricting to smooth numbers is not overwhelming when already considering the complete Vinogradov-type system.

After obtaining some preliminary admissible exponents, one can apply the method of Baker [1, Chapter 4], to generate Weyl-type estimates on a set of minor arcs in $[0,1]^{k}$. By employing a suitable Hardy-Littlewood dissection, these estimates can be used in conjunction with the preliminary Vinogradov exponents and standard major arc information to show that $\eta(s, k)=0$ and $\Delta(s, k)=0$ are admissible whenever $s$ is sufficiently large in terms of $k$. Improved admissible exponents can then be calculated by further iterating the above lemmas.

LEMma 4.3. Suppose that $\Delta(s, k-1)$ is admissible for $(1.3)$, and let

$$
\tau(k)=\max _{(k-1) \mid s} \frac{1-2 \Delta(s, k-1)}{4 s} .
$$

If $|f(\boldsymbol{\alpha})| \geq P^{1-\beta(k)}$, where $\beta(k)<\max \left\{2^{1-k}, \tau(k)\right\}$, then there exist integers $q, a_{1}, \ldots, a_{k}$ with $\left(q, a_{1}, \ldots, a_{k}\right)=1$ such that

$$
q \leq P^{1 / k} \quad \text { and } \quad\left|q \alpha_{j}-a_{i}\right| \leq P^{1 / k-j} \quad(1 \leq j \leq k) .
$$

Proof. This follows from Theorem 5.1 and the proof of Theorems 4.3 and 4.4 in Baker [1] upon reversing the roles of the variables in the Weyl shift.

Let $\mathfrak{M}$ denote the subset of $[0,1]^{k}$ for which there exist $q$ and a with $\left(q, a_{1}, \ldots, a_{k}\right)=1$ satisfying $(4.1)$. Then a standard major arc analysis along the lines of Vaughan [7, Chapter 7] (see also [8, Lemma 9.2], and [6, Lemmas 3.2 and 3.3]) establishes the following.

LEMMA 4.4. Whenever $s \geq \frac{1}{2} k(k+1)$, one has

$$
\int_{\mathfrak{M}}|f(\boldsymbol{\alpha})|^{2 s} d \boldsymbol{\alpha} \ll P^{2 s-k(k+1) / 2+\varepsilon},
$$

and whenever $s>\frac{1}{2} k(k+1)$, one has

$$
\int_{\mathfrak{M}}|f(\boldsymbol{\alpha})|^{2 s} d \boldsymbol{\alpha}=c(s, k) P^{2 s-k(k+1) / 2}\left(1+O\left(P^{-\delta(k)}\right)\right)
$$

for some real numbers $c(s, k)$ and $\delta(k)>0$. 
Here the number $c(s, k)$ is the product of the expected singular integral and singular series, and its positivity actually follows directly from (4.2) in view of the elementary lower bound $J_{s, k}(P) \gg P^{2 s-k(k+1) / 2}$, provided that $s$ is large enough to show that the contribution from the minor arcs has smaller order of magnitude.

After infusing smooth mean values into Lemma 4 of Wooley [11], we are finally able to describe some small improvements on existing Weyl exponents for $k \geq 11$.

THEOREM 4.5. Suppose that there are coprime integers $a$ and $q$ with $P \leq q \leq P^{k-1}$ such that $\left|q \alpha_{k}-a\right| \leq q^{-1}$. Then $|f(\boldsymbol{\alpha})| \ll P^{1-\sigma(k)+\varepsilon}$, where $\sigma(k) \geq 1 / \rho_{1}(k)$ and $\rho_{1}(k)$ is given in Table 4.1.

Table 4.1. Weyl exponents and the asymptotic formulas

\begin{tabular}{rrrcr}
\hline$k$ & \multicolumn{1}{c}{$\rho_{1}(k)$} & $H_{1}(k)$ & $\rho_{2}(k)$ & $H_{2}(k)$ \\
\hline 11 & 743.409 & 706 & 1489.646 & 504 \\
12 & 999.270 & 873 & 2027.215 & 661 \\
13 & 1223.475 & 1049 & 2642.870 & 803 \\
14 & 1420.574 & 1231 & 3232.507 & 955 \\
15 & 1632.247 & 1431 & 3834.340 & 1120 \\
16 & 1856.535 & 1645 & 4501.372 & 1299 \\
17 & 2114.819 & 1879 & 5209.886 & 1492 \\
18 & 2436.255 & 2134 & 5988.000 & 1699 \\
19 & 2779.680 & 2410 & 6815.154 & 1922 \\
20 & 3150.605 & 2701 & 7705.730 & 2158 \\
\hline
\end{tabular}

When $r=1$, Corollary 1.2 suffices to cover a complete set of minor arcs, and the resulting estimate turns out to be optimal when $11 \leq k \leq 13$. As in the work of Ford [3], $r=2$ is optimal for $14 \leq k \leq 20$. Further computations reveal that $r=2$ continues to be optimal up to $k=24$, while $r=3$ becomes optimal starting at $k=25$. Combining these values with Lemma 5.4 of Ford [3], we obtain the upper bounds $\widetilde{G}(k) \leq H_{1}(k)$ for the values of $H_{1}$ recorded in Table 4.1.

Admissible values for the $\beta(k)$ arising in Lemma 4.3 satisfy $\beta(k) \geq$ $1 / \rho_{2}(k)$ for the values of $\rho_{2}$ recorded in Table 4.1. These may then be used in conjunction with the Vinogradov exponents and Lemma 4.4 to produce asymptotic formulas for $J_{s, k}(P)$ as in the argument of Wooley [12, Theorem 3]. Let $\widetilde{F}(k)$ denote the least integer $s$ for which $J_{s, k}(P)$ has an asymptotic formula of the shape given on the right-hand side of (4.2). Then one has the upper bounds $\widetilde{F}(k) \leq H_{2}(k)$ for the values of $H_{2}$ recorded in Table 4.1. 
Acknowledgements. The author thanks Craig Spencer, Bob Vaughan, and Trevor Wooley for helpful discussions.

This research was supported by a National Security Agency Young Investigator Grant (MSPF-07Y-221).

\section{References}

[1] R. C. Baker, Diophantine Inequalities, Clarendon Press, Oxford, 1986.

[2] E. Bombieri, On Vinogradov's mean value theorem and Weyl sums, in: Automorphic Forms and Analytic Number Theory, Univ. de Montréal, Montréal, 1990, 7-24.

[3] K. B. Ford, New estimates for mean values of Weyl sums, Internat. Math. Res. Notices 1995, 155-171.

[4] L.-K. Hua, Additive Theory of Prime Numbers, Amer. Math. Soc., Providence, RI, 1965.

[5] N. M. Korobov, Weyl's estimates of sums and the distribution of primes, Dokl. Akad. Nauk SSSR 123 (1958), 28-31 (in Russian).

[6] S. T. Parsell, On simultaneous diagonal inequalities, III, Quart. J. Math. 53 (2002), 347-363.

[7] R. C. Vaughan, The Hardy-Littlewood Method, 2nd ed., Cambridge Univ. Press, Cambridge, 1997.

[8] T. D. Wooley, On simultaneous additive equations II, J. Reine Angew. Math. 419 (1991), 141-198.

[9] —, On Vinogradov's mean value theorem, Mathematika 39 (1992), 379-399.

[10] -, Quasi-diagonal behaviour in certain mean value theorems of additive number theory, J. Amer. Math. Soc. 7 (1994), 221-245.

[11] —, New estimates for Weyl sums, Quart. J. Math. Oxford Ser. (2) 46 (1995), 119127.

[12] - Some remarks on Vinogradov's mean value theorem and Tarry's problem, Monatsh. Math. 122 (1996), 265-273.

[13] -, On exponential sums over smooth numbers, J. Reine Angew. Math. 488 (1997), 79-140.

Department of Mathematics and Actuarial Science

Butler University

4600 Sunset Ave.

Indianapolis, IN 46208, U.S.A.

E-mail: sparsell@butler.edu 